\title{
Annotation Guidelines for Narrative Levels
}

Adam Hammond

Adam Hammond, University of Toronto

Peer-Reviewer: Tom McEnery

Dataverse DOI: https:doi.org/10.7910/DVN/2YQVM6

Article DOI: https://doi.org/10.22148/001c.30704

ABSTRACT

These guidelines present a minimalist set of instructions for annotating narrative levels. They strive for clarity and brevity, with a focus on clear examples and helpful rules of thumb. They present a one-sentence definition of a narrative and introduce the "Let me tell you a story" rule of thumb for determining whether a narrative level boundary has been crossed. Only four attributes are introduced: narrative number, narrative level, narrator ID, and whether a given narrative is left "open" or is closed.

\section{OVERVIEW}

A set of narrative texts are to be annotated for narrative levels. Any span of text containing a narrative is to be marked with the nframe category marker. For the purpose of our task, a narrative is defined as a representation of a story (an event or series of events) by a narrator. ${ }^{1}$ The texts in our annotation set may contain a single narrative (and thus a single nframe category) or may contain multiple narratives embedded within one another (nframe categories within nframe categories). If you come to a point in a text where you are uncertain whether to indicate a shift in narrative levels, imagine inserting the phrase "Let me tell you a story" right after the proposed division point. If the phrase fits, you should likely mark a new narrative level.

The nframe category has three necessary and one optional attributes.

Journal of Cultural Analytics 6 (4). 2021. 187-193. 


\section{num attribute}

The num attribute keeps track of the identity of distinct narratives. It is needed to indicate situations in which a particular narrative is interrupted and then later resumed. The identity of a narrative is indicated by numbers. The first narrative you encounter should be numbered " 1 ", the second " 2 ", the third " 3 ," and so on.

\section{level attribute}

The level attribute is used to express the degree of embedding of a narrative. If the narrative is not embedded within any others, it is a top-level or first-degree narrative and should be given the attribute value of " $\mathrm{A} "$. . A narrative embedded within an "A"-level narrative - a narrative within a narrative, or second-degree narrative - is given the attribute value of "B". A narrative embedded within a " $\mathrm{B}$ "level narrative - a narrative within a narrative within a narrative, or third-degree narrative - is given the attribute value "C", and so on.

Note that a text may contain multiple narratives at each level. For instance, the Thousand and One Nights contains hundreds (in some tellings, exactly 1,001) of "B"-level narratives, some of which contain "C"-level narratives of their own. A simpler situation — with three "B"-level narratives, one containing a " $\mathrm{C}$ "-level narrative — is illustrated below:

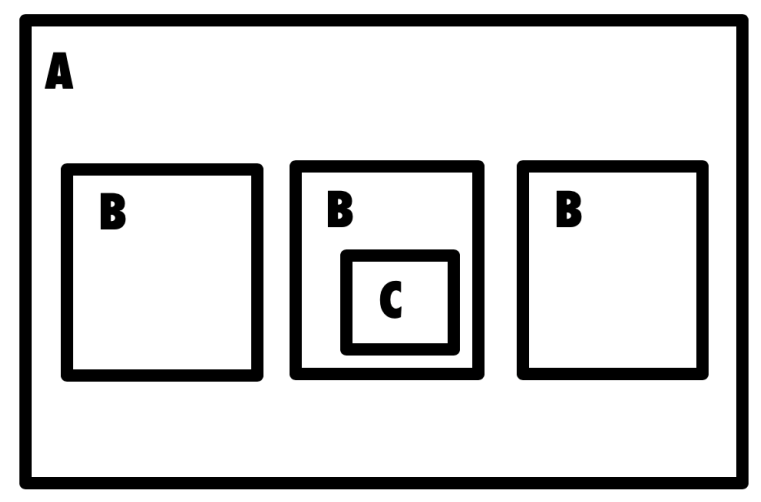




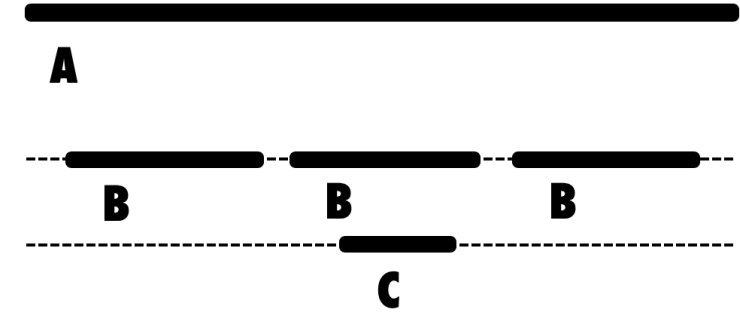

The narrative levels, or degrees of narrative, in the above structure can also be visualized as follows:

\section{narrator attribute}

The narrator attribute keeps track of the narrator who conveys the narrative. We will represent these with numbers. The first narrator you encounter should be numbered " 1 ", the second " 2 ", the third " 3 ," and so on. If the narrator of a "B"-level narrative is the same as the narrator of the " $\mathrm{A}$ " level, both are numbered " 1 ". If the narrator of a " $\mathrm{B}$ "-level narrative is different from the narrator at the " $\mathrm{A}$ " level, the first is numbered " 1 " and the second " 2 ." And so on.

\section{open attribute}

Some writers choose deliberately to leave frames "open." For our purposes, an "open" frame is one that lacks "closure," which in turn can be defined as a clear "sense of an ending." As Barbara Herrnstein Smith elaborates, closure is a narrative situation in which "stasis, or the absence of further continuation, [is] the most probable event" (Poetic Closure 34). An "open" frame is one that lacks this sense of stasis.

In Henry James's The Turn of the Screw, the governess's "C" $\mathrm{C}$ "-level tale is framed within a Christmas fireside storytelling session by two narrators, the "A"-level "I" 
and the "B"-level Douglas. Yet after the governess finishes her tale, James does not return to the "A" or "B" levels to explicitly close them. On these levels, stasis is not the most probable event: we want to know what happens to Douglas and to the first-person narrator, but we are not told. Each of these levels thus lack a "sense of an ending"; the situation is left hanging. They should thus be indicated as "open frames." Make this indication by setting the "open" attribute to "true" (if an "open" attribute is not provided, it will be assumed that the frame is "closed").

\section{SAMPLE ANNOTATIONS}

A simple text containing only one narrative might be annotated as followed, using XML markup as an example:

(1) <nframe num="1" level="A" narrator="1"> It was a dark and stormy night. The wind blew and the wolf howled. The wind blew open my window and the wolf entered. The wolf bit me and I died. $</$ nframe $>$

A text containing a single "B"-level narrative might be annotated as follows. (Since the narrator of the " $B$ "-level narrative is different from that of the " $A$ "-level narrative, it is given the narrator attribute of "2".)

(2) <nframe num="1" level="A" narrator="1"> It was a dark and stormy night. The wind blew and the wolf howled. The wind blew open my window and the wolf entered. The wolf opened his mouth and spoke. <nframe num="2" level="B" narrator="2"> "Once upon a time, when I was but a young pup, a wizard appeared before me and predicted my fate. He told me that one day, I would leap through a window and eat a man whole. After enduring many hardships, I have come to enact my fate." $</$ nframe $>$ He bit me and I died. $</$ nframe $>$ 
If the "B"-level narrative were interrupted briefly by the "A"-level narrative, indicate its continuation by marking separate nframe tags as both conveying narrative num="2":

(3) <nframe num="1" level="A" narrator="1"> It was a dark and stormy night. The wind blew and the wolf howled. The wind blew open my window and the wolf entered. The wolf opened his mouth and spoke. $<$ nframe num="2" level="B" narrator="2" > "Once upon a time," $</$ nframe $>$ the wolf said, <nframe num="2" level="B" narrator=" 2" > "when I was but a young pup, a wizard appeared before me and predicted my fate. He told me that one day, I would leap through a window and eat a man whole. After enduring many hardships, I have come to enact my fate." </nframe $>$ He bit me and I died. $</$ nframe $>$

A text containing two "B"-level narratives and a single " $\mathrm{C}$ "-level narrative might be tagged as follows. (Since the narrator of the second " $\mathrm{B}$ "-level narrative is the same as the "A"-level narrative, they share the narrator attribute of " 1 ".)

(4) <nframe num="1" level="A" narrator="1"> It was a dark and stormy night. The wind blew and the wolf howled. The wind blew open my window and the wolf entered. The wolf opened his mouth and spoke. <nframe num="2" level="B" narrator="2"> "Once upon a time, when I was but a young pup, a wizard appeared before me and predicted my fate. The wizard told me, <nframe num="3" level="C" narrator="3"> 'I was born in the east. My father was a plumber and my mother an auto mechanic. From a young age, it was clear that I had little talent for either profession, so I set off for the wizard academy. My expert wizardry has brought me here to you. You, dear wolf, will some day leap through a window and eat a man whole.' 
$</$ nframe $>$ And so here I am. After enduring many hardships, I have come to eat you." $</$ nframe $>$ Before he had a chance to eat me, I tried to distract him with a story. <nframe num="4" level="B" narrator="1"> "Once upon a time and a very good time it was there was a moocow coming down along the road and this moocow that was coming down along the road met a nicens little boy named baby tuckoo..." $</$ nframe $>$. But he found the story boring and so he bit me and I died. $</$ nframe $>$

\section{Special Case: "Open Frames"}

In the previous example, the phrase "But he found the story boring and so he bit me and I died" provides closure to the "A"-level narrative. In the following example, however, the " $\mathrm{A}$ "-level narrative is not explicitly closed by narrator 1 (presumably because he has been eaten and is unable to write). As such, the "open" attribute has been set to "true."

(5) <nframe num="1" level="A" narrator="1" open="true"> It was a dark and stormy night. The wind blew and the wolf howled. The wind blew open my window and the wolf entered. The wolf opened his mouth and spoke. <nframe num="2" level="B" narrator="2"> "Once upon a time, when I was but a young pup, a wizard appeared before me and predicted my fate. He told me that one day, I would leap through a window and eat a man whole. After enduring many hardships, I have come to enact my fate." $</$ nframe $></$ nframe $>$

\section{Special Case: "Mise-en-Abyme” Narratives}

Some narratives, especially common among postmodern authors, paradoxically embed a story within itself. This paradoxical situation can be represented by showing a series of "A"-level narratives with the same num attribute embedded within 
one another:

(6) <nframe num="1" level="A" narrator="1"> It was a dark and stormy night. The band of robbers huddled together around the fire. When he had finished eating, the first bandit said, <nframe num="1" level="A" narrator="1"> "Let me tell you a story. It was a dark and stormy night and a band of robbers huddled together around the fire. When he had finished eating, the first bandit said: <nframe num="1" level="A" narrator="1"> 'Let me tell you a story. It was a dark and stormy night and...'" $</$ nframe $></$ nframe $></$ nframe $>$

\section{OTHER NOTES}

If a shift in narrative level occurs around a chapter break and you're unsure whether to put your nframe category marker before or after the chapter header, put it after.

\section{Notes}

\footnotetext{
${ }^{1}$ This definition is derived from Porter Abbott, The Cambridge Introduction to Narrative, 2nd ed. (Cambridge: Cambridge University Press, 2014), 237, where narrative is defined as "the representation of a story (an event or series of events)".

${ }^{2}$ The notion of degrees of narrative employed in this annotation derives from the work of Shlomith Rimmon-Kenan as discussed in Manfred Jahn, "N2.4 Narrative Levels," in Narratology: A Guide to the Theory of Narrative (Köln, 2017).
} 